Décadrages Décadrages

cınéma, à travers champs Cinéma, à travers champs

34-36 | 2017

Cinéma de re-montage

\title{
Joseph Cornell : le film de re-montage et la séance cinématographique comme forme de ready-made aidé
}

\section{François Bovier}

\section{(2) OpenEdition \\ Journals}

Édition électronique

URL : https://journals.openedition.org/decadrages/1068

DOI : 10.4000/decadrages. 1068

ISSN : 2297-5977

\section{Éditeur}

Association Décadrages

\section{Édition imprimée}

Date de publication : 1 mai 2017

Pagination : 96-121

ISBN : 978-2-9700963-3-7

ISSN : 2235-7823

\section{Référence électronique}

François Bovier, « Joseph Cornell : le film de re-montage et la séance cinématographique comme forme de ready-made aidé », Décadrages [En ligne], 34-36 | 2017, mis en ligne le 19 août 2019, consulté le 04 avril 2022. URL : http://journals.openedition.org/decadrages/1068 ; DOI : https://doi.org/ 10.4000/decadrages.1068 


\section{Joseph Cornell : le film de re-montage et la séance cinématographique comme forme de ready-made aidé}

1 Sur les origines du re-montage dans le cinéma soviétique, où cette pratique se généralise, voir Lev Kouléchov, «Le remontage» [I920], L'Art du cinéma et autres écrits (François Albera [éd.]), Lausanne, L'Age d'Homme, I990, pp. 64-67; ce texte, inédit du vivant de Kouléchov, est partiellement repris dans «La bannière du cinématographe» [1920], dans L. Kouléchov, op. cit., pp. 36-63. Kouléchov écrit notamment: «A la tête d'une des grandes sociétés cinématographiques, V. R. Gardine s'était trouvé devant la nécessité de composer des films uniquement à partir de fragments déjà filmés, sans scénarios, sans intertitres, et sans que les scènes soient numérotées dans l'ordre littéraire du scénario. Bien entendu, dans ce cas, V. R. Gardine cherchait, en assemblant ces films, à obtenir un résultat dans la composition, et devait structurer l'ensemble du film uniquement par le montage en faisant fi du scénario original.» (Id., pp. 64-65.) Kouléchov est nommé chef de la section de re-montage des films étrangers pour le VFKO en I9I9. La pratique du re-montage n'en attire pas moins un certain nombre de critiques. Tynianov peut ainsi écrire: «Dans La Rue sans joie [Die freudlose Gasse, G. W. Pabst, I925], au lieu de l'héroïne, c'est du fiancé qu'on fait l'assassin. Le re-monteur devait être content de son travail. Mais les mains de l'assassin

Les films de Joseph CoRnell, ou plutôt Rose Hobart (I936) qui en vient à représenter «métonymiquement» l'ensemble de son corpus cinématographique - pourtant varié, hétéroclite et comprenant également des films expressément tournés à sa demande -, sont investis comme l'acte inaugural du cinéma de found footage. Je reviendrai sous peu sur ce moment «fondateur», et sur le contexte artistique dans lequel il convient de l'inscrire (à savoir l'importation du surréalisme aux Etats-Unis, notamment par le biais d'expositions). Mais auparavant, je ferai brièvement retour, dans une perspective historiographique, sur les pratiques du cinéma de re-montage et du found footage - qui peuvent être articulées sans pour autant effacer ou nier leurs spécificités.

Le cinéma de «re-montage», comme l'indique le préfixe itératif «re-», exacerbe voire autonomise la fonction du montage dans l'acte de création: il s'agit là d'une forme de montage second ou, si l'on préfère, d'un geste de réassemblage de plans préexistants (dont le sens est mouvant, dépendant étroitement de leur articulation entre eux), suivant un processus de décontextualisation et recontextualisation des images. En URSS, dans les années I920, il était usuel de remonter des films étrangers afin d'en modifier le sens idéologique, notamment en insérant des intertitres ${ }^{1}$. Dans le contexte du cinéma documentaire soviétique, l'enjeu du re-montage pouvait être de reconstituer un événement historique à partir de documents d'archives soigneusement sélectionnés (notoirement dans La Chute de la dynastie Romanov, I927, d'Esfir Choub, après

sur l'écran restent celles d'une femme. [...] C'est là un cas de «re-montage artistique, car il est difficile de lui trouver des motivations idéologiques. Dans les films bien faits, l'idéologie ressort à tel point par des éléments du sujet et du style que les ciseaux ni la colle n'y peuvent rien.» (Iouri Tynianov, «Sur le re-montage» [1926], dans François Albera [éd.], Les Formalistes russes et le cinéma: Poétique du film, Paris, Nathan, 1996, p. 199). 
visionnement de 60 o०० mètres d'archives tournées entre I9I2 et I9I7) ${ }^{2}$; ou, à l'opposé, le propos pouvait être d'interpeller le public par la puissance de disruption du montage, suivant le programme de l'agit-prop ${ }^{3}$. Pour désigner cet ensemble de pratiques, le terme qui s'impose dans la littérature secondaire est celui de «compilation»: comme l'a montré de façon convaincante Jay Leyda ${ }^{4}$, le «film de compilation» constitue si ce n'est un genre cinématographique à part entière, tout au moins un geste récurrent, en lien avec une intention politique ou une volonté de propagande. Le matériau de départ, souvent issu d'actualités, est remis en scène, ré-agencé ou ré-ouvré, dans une structure déterminée, suivant une perspective qui recoupe la théorie vertovienne de la «fabrique du fait» ${ }^{5}$, ou plus largement la notion de «document». C'est en vain que l'on s'attacherait à déceler une quelconque velléité artistique dans ces films de re-montage, ou alors il faut l'entendre exclusivement dans le sens avant-gardiste et utopique d'une dissolution de l'œuvre dans le champ socio-politique (à l'instar de ce qui se joue dans le productivisme russe, les artistes produisant des objets et non plus des artefacts esthétiques).

Par la suite, les études sur le re-montage tendent à se focaliser sur le cinéma expérimental et les films d'artistes, relativisant par là-même la dimension éminemment politique du «cinéma de compilation»; le terme de ralliement, cette fois, est le «found footage» ${ }^{6}$. Sa parenté avec

2 Esfir Choub travaille dès I922 au Goskino comme re-monteuse de films destinés au public soviétique; dans les années I930, elle réalise plusieurs films de re-montage centrés sur des événements historiques. Voir Albera supra et Alexandre Sumpf, Révolutions russes au cinéma. Naissance d'une nation: URSS, I9I7I985, Paris, Armand Colin, 20I5, pp. 77-84.

3 Voir François Albera, "Cinéma soviétique des années 1924-I928: le film de montage/document, matériau, point de vue», dans Jean-Pierre Bertin-Maghit (éd.), Une histoire mondiale des cinémas de propagande, Monts, Nouveau Monde éditions, 2008, pp. 83-9I; Yuri Tsivian, «The Wise and Wicked Game: Re-editing and Soviet Film Culture of the I920s », Film
History, vol. 8, n 3, I996, pp. 327-343. Ces pratiques (re-montage d'actualités, films d'agit-prop, etc.) se déploient également en Europe, sous l'égide du Secours ouvrier international (SOI), coordonné par Willy Münzenberg (voir son ouvrage manifeste: Erobert den Film!, Berlin, Neuer Deutscher Verlag, I925). Sur le SOI, voir Kasper Braskén, The International Workers' Relief, Communism, and Transnational Solidarity. Willy Münzenberg in Weimar Germany, Houndmills/ New York, Palgrave Macmillan, 2015.

4 Jay Leyda, Film Beget Films: A Study of the Compilation Film, New York, Hill and Wand, i964. Voir aussi Jaimie Baron, The Archive Effect: Found Footage and the Audiovisual Experience of History, New
Jersey, Routledge, 20I4; Patrik Sjöberg, The World in Pieces: A Study of Compilation Films, Stockholm, Aura Förlag, 200I.

5 Voir Dziga Vertov, «La fabrique des faits (à titre de proposition)» [Pravda, 24 juillet I926], Articles, journaux, projets, Paris, UGE, I972, pp. 83-86.

6 Voir Eugeni Bonet (éd.), Desmontaje: Film, Vídeo/Apropriación, Reciclaje, Valence, IVAM, I993; Peter Tscherkasski (éd.), "Found Footage. Filme aus gefundenem Material», Blimp, n ${ }^{\circ} 6$, printemps I99I; William C. Wees, Recycled Images: The Art and Politics of Found Footage Film, New York, Anthology Film Archives, I993. Voir également les catalogues d'exposition suivants: Yann Beauvais et Danièle Hibon (éd.), Found Footage: analyse, collage, mélancolie, Paris, Editions du Jeu de Paume, I995; Marente Bloemheuvel, Giovanna Fossati et Jaap Guldemond (éd.), Found Footage: Cinema Exposed, Amsterdam, Eyefilm/Amsterdam University Press, 2012; Cecilia Hausheer et Christoph Settele (éd.), Found Footage Film, Lucerne, Viper/Zyklop Verlag, I992. Le terme de «found footage», qui est employé concurremment avec d'autres expressions ("archival footage», «stock footage», «recycled footage», «collage film»), se généralise avec un certain décalage par rapport aux pratiques filmiques, les figeant ainsi à travers des traits définitoires. Les enjeux liés à ces pratiques sont déjà débattus dans les années i950, à travers la notion de détournement (voir Guy-Ernest Debord et Gil J. Wolman, «Mode d'emploi du détournement», Les Lèvres nues, nº 8, mai i956, pp. 2-9). Notons encore la fondation récente de la revue Found Footage Magazine, dont le premier numéro (octobre 2015) est consacré à Bill Morrison, et le deuxième (mai 20ı6) à Barbara Hammer. 
7 André Breton, «Crise de l'objet» [Cahiers d'art, $\mathrm{n}^{\circ}$ I-2, I936, pp. 2I-28], Le Surréalisme et la peinture, Paris, Gallimard, I965, p. 359. Sur ce point, voir également André Breton, «Equation de l'objet trouvé» [Documents 34, n I, juin I934, pp. I7-24], L'Amourfou, Paris, Gallimard, I976 [ı937], pp. 38-5i.

8 Voir Pierre Reverdy, «L'image» [Nord-Sud, n ${ }^{\circ}$ 13, mars I9I8], Nord-Sud, Self defence et autres écrits sur l'art et la poésie, Paris, Flammarion, 1975, pp. 7375. Reverdy écrit notamment: «L'image est une création pure de l'esprit. Elle ne peut naître d'une comparaison, mais du rapprochement de deux réalités plus ou moins éloignées. Plus les rapports de deux réalités rapprochées seront lointains et justes, plus l'image sera forte - plus elle aura de puissance émotive et de réalité poétique.» Breton s'approprie cette citation dans le premier Manifeste du surréalisme (Paris, Le SagittaireSimon Kra, 1924).

9 André Breton, "Crise de l'objet», op. cit., p. 355 .

10 Jacques-André Boiffard, Paul Eluard et Roger Vitrac, «Préface», La Révolution surréaliste, $\mathrm{n}^{\circ} \mathrm{I}$, décembre $\mathrm{I} 924$, p. I.

11 Joseph Cornell est intégré à la première exposition surréaliste américaine organisée par Julien Levy, en janvier I932. Sur les liens de Cornell au surréalisme, voir Sylvie Ramond et Matthew Affron (éd.), Joseph Cornell et le surréalisme à New York: Dali, Duchamp, Ernst, Man Ray, Paris, Hazan, 2013. «l'objet trouvé» surréaliste (found object) est patente, et la proximité des démarches évidente. Rappelons que pour Breton, la «démarche surréaliste» tend à «provoquer une révolution totale de l'objet», qu'il spécifie en ces termes:

«action de le détourner de ses fins en lui accolant un nouveau nom et en le signant, qui entraîne la requalification par le choix (〈ready made) de Marcel Duchamp); de le montrer dans l'état où l'ont mis parfois les agents extérieurs, tels les tremblements de terre, le feu et l'eau; de le retenir en raison même du doute qui peut peser sur son affectation antérieure, de l'ambiguïté résultant de son conditionnement totalement ou partiellement irrationnel, qui entraîne la signification par la trouvaille (objet trouvé) et laisse une marge appréciable à l'interprétation au besoin la plus active (objet trouvé-interprété de Max Ernst); de le reconstruire enfin de toutes pièces à partir d'éléments épars pris dans le donné immédiat (objet surréaliste proprement dit).» ${ }^{7}$

Breton, en s'appropriant la définition de l'image poétique de Reverdy comme «rapprochement de deux réalités plus ou moins éloignées ${ }^{8}$, prend soin de préciser que c'est contre l'usage commun de l'objet que le surréalisme se positionne:

"Cette faculté de rapprochement des deux images leur permet [aux poètes, aux artistes, aux savants] de s'élever au-dessus de la considération de la vie manifeste de l'objet, qui constitue généralement une borne. Sous leurs yeux, au contraire, cet objet, tout achevé qu'il est, retourne à une suite ininterrompue de latences qui ne lui sont pas particulières et appellent sa transformation.» ${ }^{9}$

C'est déjà ce credo du détournement de la fonctionnalité de l'objet que revendiquent Jacques-André Boiffard, Paul Eluard et Roger Vitrac dans leur préface au premier numéro de La Révolution surréaliste, en I924:

«Toute découverte changeant la nature, la destination d'un objet ou d'un phénomène constitue un fait surréaliste. ${ }^{10}$

$\mathrm{Au}$ vu de ces rapprochements, qui ne sont pas que lexicaux, il n'est guère surprenant que dans les études portant sur le cinéma de found footage une place de choix soit accordée à Joseph Cornell: ce dernier, étroitement associé au surréalisme aux Etats-Unis ${ }^{11}$ - malgré ses réserves par 
rapport au mouvement fédéré par Breton ${ }^{12}$ - transpose dans le champ du film la technique du ready-made aidé ${ }^{13}$. Quant à son œuvre d'assemblagiste, à travers laquelle il acquiert une certaine reconnaissance critique, elle s'inscrit résolument dans la poétique de l'«objet trouvé», notamment dans sa relation aux collages de Max Ernst (La Femme roo têtes ${ }^{14}$ lui sert d'inspiration pour ses premières œuvres). Ses collages et ses boîtes - souvent vitrées - exposent divers objets récupérés (naturels ou manufacturés: des cartes ou des inscriptions d'hôtel effacées, des perroquets ou des papillons épinglés, des coquillages ou des étoiles de mer, des fioles ou des dés à coudre, du sable dans des verres brisés, etc.) qui sont parfois associés à des images de danseuses ou d'actrices. Mais à la différence des surréalistes, Cornell travaille à partir de formes culturelles déconsidérées et réputées comme féminines ou infantiles, tels les objets de vitrine, les miniatures ou le dispositif du penny arcade. Il se tient ainsi en retrait du marché de l'art, privilégiant une économie du don (il réalise ses objets, ses assemblages et ses boîtes pour des stars hollywoodiennes ou des enfants, par exemple, les soustrayant de ce fait à l'économie marchande ${ }^{15}$ ). Dans tous les cas, il manifeste une obsession pour l'iconographie des années 1920, tout en mettant en exergue l'aura du fragment. Sa conception du cinéma s'inscrit dans le prolongement direct de son travail de plasticien: dans ses films, il combine des images disparates qu’il a récupérées, ne tournant pas lui-même les plans (quand il «tourne»effectivement des films, situés dans l'époque contemporaine, il fait appel à des photographes et cinéastes indépendants, à l'instar de Rudy Burckhardt, Stan Brakhage ou encore Larry Jordan ${ }^{16}$ ).

12 Cornell, à l'occasion de l'exposition Fantastic Art, Dada, Surrealism qui s'ouvre en décembre 1936 au MoMA, demande au commissaire Alfred H. Barr Jr. d'être présenté dans le catalogue comme un «constructiviste américain» et non comme un «surréaliste» (Lindsay Blair, Joseph Cornell's Vision of Spiritual Order, Londres, Reaktion Books, I998, p. 32). Dans une lettre au directeur du MoMA, Cornell se distancie explicitement du surréalisme: «Je ne partage pas les théories du rêve et de l'inconscient des surréa- listes. Bien que je sois un fervent admirateur de la plupart de leurs œuvres, je n'ai jamais été officiellement un surréaliste, et je pense que le surréalisme présente des potentialités qui n'ont pas été développées jusqu’à présent» (cité par Erika Doss, «Joseph Cornell and Christian Science», dans Jason Edwards et Stephanie L. Taylor [éd.], Joseph Cornell: Opening the Box, Oxford/Berne, Peter Lang, 2007, p. I26; ma traduction). Le caractère sulfureux du surréalisme et son apologie de l'inconscient entrent en contradiction avec le goût de Cornell pour la discrétion et son implication dans la Science chrétienne.

13 Voir L.H.O.O.Q. (I9I9) de Marcel Duchamp, prototype du ready-made aidé: le titre (lire: «elle a chaud au cul» ou, alternativement, «look») apposé à une reproduction de La Joconde de Léonard de Vinci ainsi que la moustache et le bouc ajoutés à la jeune femme résolvent l'énigme de son sourire. D'après Breton, «Les ready made et ready made aidés, objets choisis ou composés, à partir de I9I4, par Marcel Duchamp, constituent les premiers objets surréalistes» (André Breton et Paul Eluard, Dictionnaire abrégé du surréalisme [Galerie des Beaux-Arts, I938], Paris, José Corti, 2005, p. I8). De 1942 à I946, Cornell travaille comme assistant pour Duchamp (qu’il a rencontré en I935), lorsque celui-ci est établi à New York. La Boîte en valise (1942-1946), musée portatif qui réplique en miniature les œuvres de Duchamp, exerce une influence profonde sur les boîtes de Cornell.

14 Voir Max Ernst, La Femme Ioo têtes, Paris, Editions du Carrefour, I929.

15 Voir Dickran Tashjian, Joseph Cornell: Gifts of Desire, Miami Beach, Grassfield Press, i992.

16 Cornell a réalisé plusieurs films sur des espaces urbains et des parcs (Bookstalls, circa 1938-1939; The Aviary, I955, avec Burckhardt; Centuries of June, avec Brakhage, I955; Joanne, Union Square, I955, avec Burckhardt; What Mozart Saw on Mulberry Street, 1956, avec Burckhardt; A Legend for Fountains, I957, avec Burckhardt; Nymphlight, I957, avec Burckhardt; Angel, I957; Mulberry Street, I95765, avec Burckhardt; Flushing Meadows, I965, avec Jordan), ou encore des lieux en voie de destruction (Gnir Rednow, I955, avec Brakhage). 
17 Voir Adam Lowenstein, Dreaming of Cinema: Spectatorship, Surrealism, and the Age of Digital Media, New York, Columbia University Press, 2015, Pp. 149I52; P. Adams Sitney, Visionary Film: The American Avant-Garde, Oxford, Oxford University Press, I974, pp. 386387 ; P. A. Sitney, The Cinema of Poetry, Oxford, Oxford University Press, 2015, pp. II3-I38.

18 Voir Christa Blümlinger, Cinéma de seconde main. Esthétique du remploi dans l'art du film et des nouveaux médias, Paris, Klincksieck, 2013, pp. 35-36 [première publication: Kino aus zweiter Hand. Zur Ästhetik materieller Aneignung im Film und in der Medienkunst, Berlin, Vorwerk 8, 2009]; Jeffrey Skoller, Shadows, Specters, Shards: Making History in Avant-Garde Film, Minneapolis/ Londres, University of Minnesota Press, 2005, pp. 7-8; William C. Wees, Recycled Images, op. cit., p. 9. Michael Pigott fait écho à ce point de vue: si, selon lui, Rose Hobart n'est pas le «premier film de found footage» à être réalisé, il constitue cependant «le premier film de collage incontournable» [the most prominent early collage film] (M. Pigott, Joseph Cornell versus Cinema, Londres, Bloomsbury, 2013, p. 9).

\section{Rose Hobart, ou la poétique de l'objet trouvé}

Assigner l'origine de l'usage artistique du cinéma de re-montage à Joseph Cornell, avec la «réalisation» de Rose Hobart (i936), constitue un lieu commun dans la littérature secondaire consacrée au cinéma expérimental ${ }^{17}$ et au found footage ${ }^{18}$. Rappelons les faits et leur contexte. Cornell assemble son film à partir de plans extraits d'East of Borneo (I93I,

Rose Hobart (Joseph Cornell, 1936)
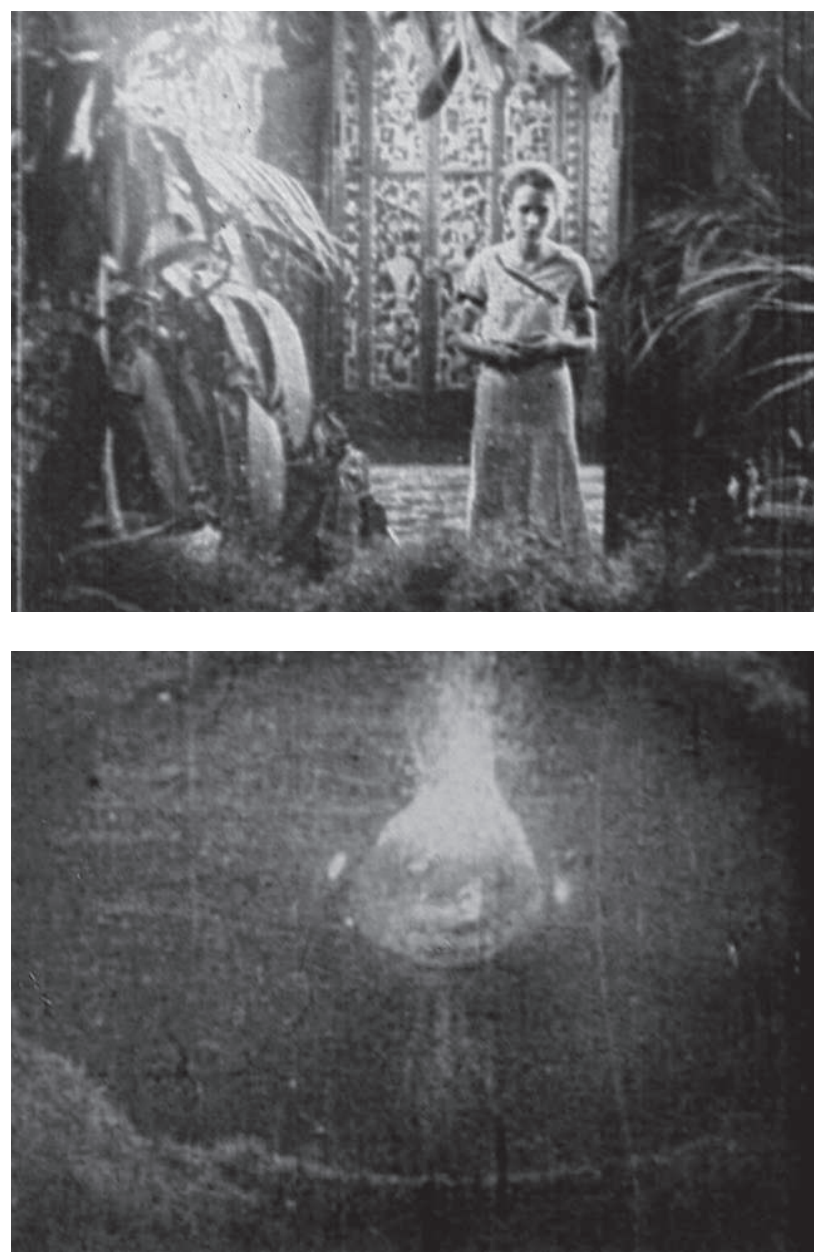
George Melford), un mélodrame exotique produit par Universal Pictures, tout en incorporant dans cet ensemble relativement homogène ${ }^{19}$ des plans tirés de sa propre collection de films (notamment des films scientifiques et éducatifs). S'inscrivant dans la poétique surréaliste de «l’objet trouvé» (Cornell a acheté East of Borneo parmi un vaste lot de pellicule usagée), Cornell se concentre dans ce film sur les plans où figure l'actrice Rose Hobart; de plus, il interpose un filtre bleu à l'image, colorisation signifiant conventionnellement la nuit dans le cinéma muet, tout en ralentissant la cadence de projection du film (à ı6 images par seconde) et en substituant à la bande-son d'origine un disque 78 tours de musique brésilienne (Holiday in Brazil de Nestor Amaral) trouvé dans un magasin de seconde main à Manhattan ${ }^{20}$. La «rectification» de l'objet trouvé est orientée ici par une nostalgie pour le cinéma muet. Celle-ci, il faut le rappeler, constitue un trait récurrent chez Cornell; ce dernier peut ainsi évoquer, dans son hommage à Hedy Lamarr publié dans View, «le pouvoir étonnant qu’a le cinéma muet de suggérer un monde de beauté idéal»:

«Nous remercions donc Hedy Lamarr, la flâneuse enchantée, d’avoir parlé de nouveau le langage poétique et évocateur du muet, même si ce n'est parfois qu'un murmure à côté du rugissement vide de la bande-son...» ${ }^{21}$

Précisons encore que Rose Hobart s'ouvre sur un plan de personnes qui observent une éclipse solaire, et se referme sur la lune qui passe devant le soleil avant de revenir à l'actrice Rose Hobart qui ferme les yeux, suggérant que le film re-monté est lui-même investi comme une forme d'«éclipse» cinématographique ${ }^{22}$. De là à ériger Rose Hobart en un film qui anticipe les stratégies de recyclage et d'appropriation mises en œuvre dans les années i960 par Bruce Conner ou Arthur Lipsett, il n’y a qu’un pas vite franchi...

Comme souvent, le mythe n'est pas entièrement dépourvu de fondement: cette œuvre présente une dimension inaugurale par le caractère radicalement décousu de son montage et par un fétichisme exacerbé qui innerve la cinéphilie «classique». Le caractère insinuant et nostalgique du rythme de Rose Hobart, l'«aura» reconstruite de la star et le démembrement de son corps par le montage, la forte charge symbolique associée à ses gestes et à ses mimiques, détachés de tout ancrage diégétique, sont remarquables - le film gagnerait d'ailleurs à être étudié à travers le
19 Le film de Melford, qui incorpore des plans de films animaliers (tigres, crocodiles) ou documentaires (volcan en éruption), s'écarte lui-même de la logique du cinéma de la continuité.

20 Voir notamment Chantal Le Sauze, «Le temps suspendu ou l'univers cinématique de Joseph Cornell», I895. Revue de l'Association française de recherche sur le cinéma, $\mathrm{n}^{\circ}$ 4I $^{\mathrm{I}}$ (Valérie Vignaux [éd.], "Archives»), 2013, pp. 45-55.

21 Joseph Cornell, ««Enchanted Wanderer $>$ : Excerpt from a Journey Album

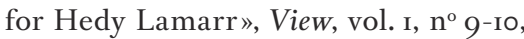
décembre I94I-janvier I942, cité dans Annette Michelson, «Joseph Cornell. Rose Hobart et Monsieur Phot: le cinéma d'Utopia Parkway», dans Germain Viatte (éd.), Peinture Cinéma Peinture, Marseille/Paris, Musées de Marseille/ Hazan, i989, p. 236.

22 A ce sujet, Erik Bullot s'interroge: «S'agit-il pour le spectateur d'observer l'ombre portée du film princeps, à la manière d'une éclipse, à travers le filtre bleu passé devant le projecteur?» (Erik Bullot, Sortir du cinéma: Histoire virtuelle des relations de l'art et du cinéma, Genève, MAMCO, 2013, p. 156). Voir aussi Jodi Hauptman, Joseph Cornell: Stargazing in the Cinema, New Haven/Londres, Yale University Press, I999, pp. IoI-I07. 
prisme de l'étude des rapports sociaux de sexe, le voyeurisme dirigé sur la jeune actrice s'inversant en fétichisme, c'est-à-dire en désaveu de la différence sexuelle (le motif récurrent de la bougie sur le point de s'éteindre ne renvoie pas seulement au scintillement du faisceau du projecteur, mais aussi au complexe de la castration). La stratégie de re-montage de Cornell, qui consiste à suspendre la signification et la finalité narrative
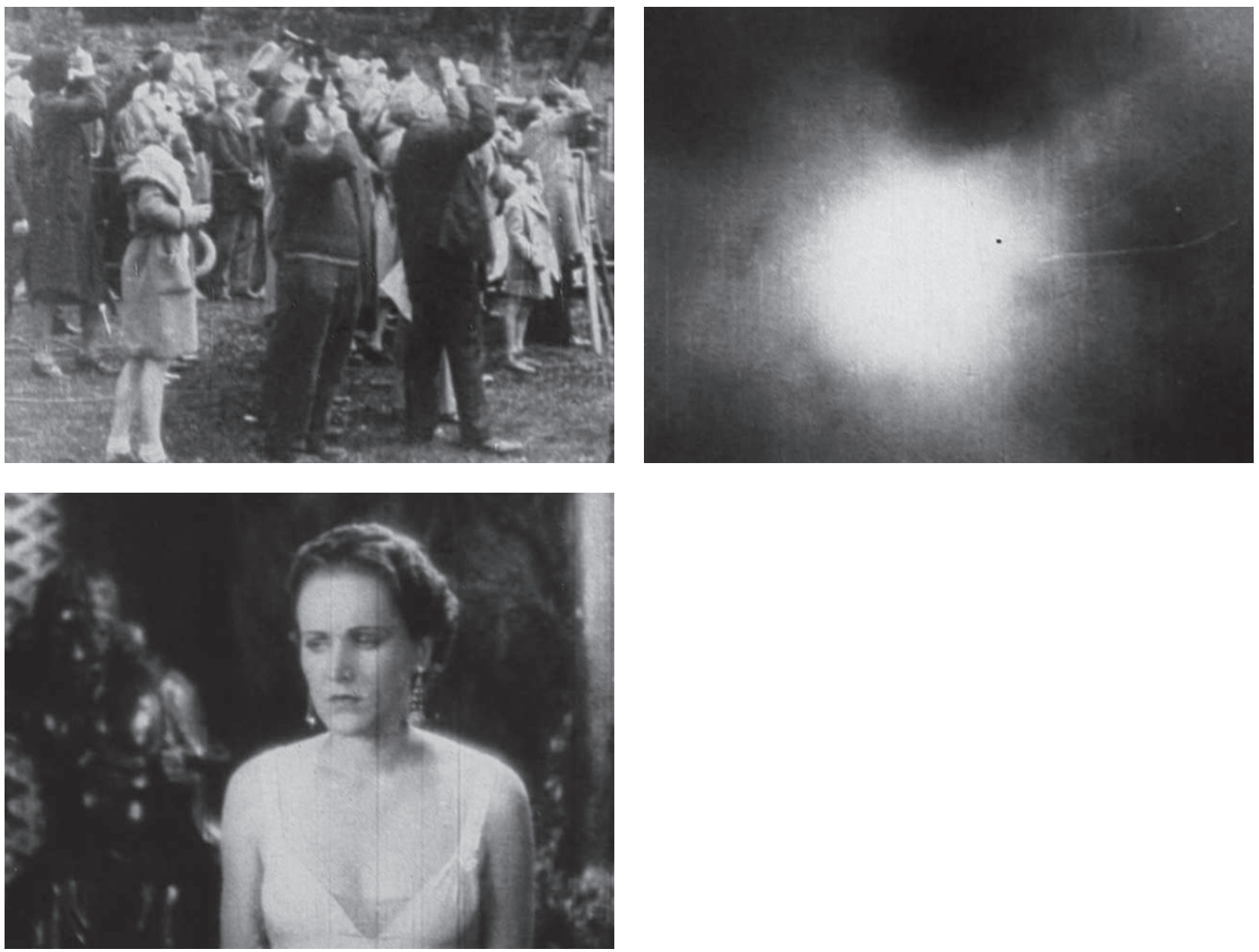
des plans, suscite une authentique tension filmique, un singulier sens du suspens: des éléments disparates, désarrimés, sont juxtaposés; la répétition de gestes, d'attitudes, de motifs plastiques et thématiques, qui donnent lieu à une série de variations, se conjoignent dans la constitution d'un portrait féminin idéalisé, en multipliant les points de vue et les perspectives. Comme souvent dans le cinéma de re-montage, Cornell a recours conjointement à des raccords de continuité (principalement sur le regard) et à des faux raccords, pour relier ou disjoindre les plans entre eux. Ainsi, le film de série B, sur le plan de sa structuration narrative, est mis en pièce; mais ce qui est révélé, c'est le potentiel d'imagerie «surréaliste» latent qui est inscrit dans un film des studios hollywoodiens, une fois libéré de sa causalité narrative. Cornell s’inscrit ainsi en faux contre la tentative «moderniste» de légitimer le cinéma en tant qu'art, que l'on peut décrire comme la volonté d'instituer un langage purement visuel à partir de l'autonomisation de ses signes iconographiques et de ses procédés de composition formelle.

Nulle équivoque ici: le re-montage de Cornell fétichise la star et non la structure formelle du film, l'attention du spectateur se focalisant sur des détails incidents du cadre et les plus infimes inflexions de l'actrice, suivant une logique psycho-symptomatologique (l'interprétation élaborée par Freud dans Psychopathologie de la vie quotidienne ${ }^{23}$ me paraissant plus probante que la méthode paranoïaque-critique de Dali ${ }^{24}$ pour appréhender la logique mise en jeu dans Rose Hobart). Il faut d'ailleurs signaler la complexité du statut de cette figure: Rose Hobart, parfois habillée en homme (une inversion constante chez Cornell, fasciné par l'ambivalence entre les sexes), constitue une figure androgyne, qui fait peutêtre écho au surnom de Duchamp, Rrose Sélavy ${ }^{25}$ (son double «féminin» qui peut se lire: «Eros, c'est la vie»). Et si l'on en croit Sitney ${ }^{26}$, Cornell aurait par la suite utilisé Tristes tropiques comme titre alternatif à Rose Hobart, signalant la parenté de ce film avec les études de Lévi-Strauss ${ }^{27}$ - et invoquant, par la même occasion, un regard ethnographique qui s'oppose à l'exotisme manifeste dans le film de Melford. J'irai jusqu'à hasarder l'hypothèse suivante: la projection fantasmatique (blanche et masculine) d’une altérité désignée comme menaçante, qui est évidente dans le film de Melford, est déconstruite dans le film de re-montage de Cornell. L'orientalisme - pour reprendre ce concept théorisé par Edward Saïd ${ }^{28}$ - est ici désigné en tant que tel, et non reproduit à l'identique
23 Voir Sigmund Freud, Psychopathologie de la vie quotidienne, Paris, Payot, 1922 [première publication: Zur Psychopathologie des Alltagaslebens, Berlin, Karger, I90I].

24 Voir notamment Salvador Dali, "L'âne pourri» [1930]; "Nouvelles considérations générales sur le mécanisme du phénomène paranoïaque du point de vue surréaliste» [1933]; «Psychologie non euclidienne d'une photographie» [I935], Oui. Méthode paranoïaque-critique et autres textes, Paris, Denoël, I97ı, Pp. 153I58; p. 2 II ; pp. 249-254.

25 Voir Jodi Hauptman, Stargazing in Cinema, op. cit., p. III; Thomas Lawson, «Silently, by Means of a Flashing Light», October, vol. I5, hiver I980, pp. 49-6o.

26 Voir P. Adams Sitney, Visionary Film, op. cit., p. 386.

27 Voir Claude Lévi-Strauss, Tristes tropiques, Paris, Plon, I955.

28 Voir Edward Saïd, L'Orientalisme. L'Orient créé par l'Occident, Paris, Seuil, I980 [première publication: Orientalism, Londres, Penguin, I977]. 

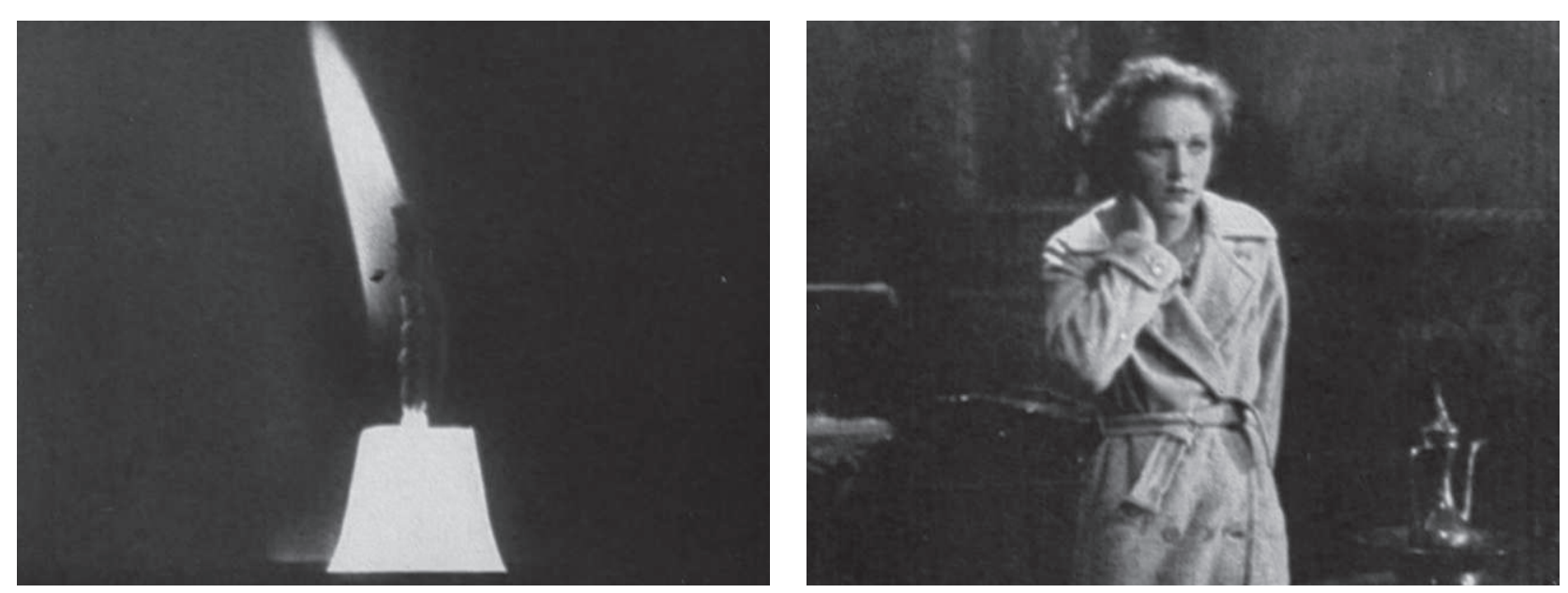

29 Voir Man Ray, Autoportrait, Paris, Laffont, I964, pp. 267-269 [première publication: Self Portrait, Boston, Atlantic Monthly Press, 1963]. Sur Le Retour à la raison, voir Deke Dusinberre, «Le Retour à la raison: sens et non-sens», dans Jean-Michel Bouhours et Patrick de Haas (éd.), Man Ray, directeur du mauvais movies, Paris, Centre Georges Pompidou, I997, pp. 28-39. suivant une logique de domination: la nostalgie qui se dégage du film peut ainsi être comprise comme une stratégie de mise à distance et d'analyse critique des images reconduites, suivant une esthétique que l'on pourrait qualifier de camp et une propension à "performer» l'altérité (sexuelle, raciale).

Malgré le mouvement d'interprétation infinie que semble appeler ce film, la fonction de précurseur de Rose Hobart est avant tout entretenue par un discours hagiographique, rituellement convoqué par Cornell, qu'il convient d'interroger, celui-ci confinant à la mystification - c'est là aussi une tradition surréaliste: que l'on songe aux récits de Man Ray sur la genèse de son film Retour à la raison (1925) ${ }^{29}$. La version la plus accréditée de ce récit des origines est due à Julien Levy, un important galeriste new-yorkais qui s'est activement impliqué dans la constitution de la New York Film Society. Dans ses mémoires, il décrit en ces termes la séance à l'occasion de laquelle Cornell projette Rose Hobart:

«Sur l'écran, l'héroöne silencieuse prononce des promesses muettes. Soudainement, un cheik pénètre dans la tente. La jeune Britannique sans défense relève la couverture de son lit contre sa poitrine, dévisageant l'intrus avec une fascination terrifiée. Elle respire profondément et crispe ses poings. Les yeux du cheik brillent avec un sentiment d'anti- 
cipation rapace. Une balle roule au ralenti et tombe dans un bassin. Le cheik, la jeune fille britannique, le cheik, des chameaux qui courent, la balle, la jeune fille à nouveau, la chute de la balle...

Tout à coup, on entend un énorme fracas. 〈Salaud!` Le silence est rompu. 〈Lumière!〉, j’ordonne. 〈Calme-toi , implore Gala qui se précipite vers Dali. «Salaud, et encore salaud `, crie Dali qui se fraie vivement un chemin vers Cornell.

Dali était furieux, un état auquel il nous a habitué mais qui était particulièrement impressionnant cette fois, oscillant entre le déchaînement d'un démon [...] et la frustration d'un enfant suite à une crise de nerf. [...] Il y avait peu de choses à dire après que Gala ait apaisé Dali et que j’aie réconforté Cornell. «Mon idée pour un film correspondait exactement à cela; et j'étais sur le point de la proposer à une personne qui allait me donner les moyens de la réaliser. Je ne peux pas dire que Cornell m’a volé mon idée`, précise Dali, «je ne n'en ai pas conservé de trace écrite et je n'en ai parlé à personne, mais c'est comme s'il l'avait volée.)

Cornell se lamentait sans fin, «Mais pourquoi, pourquoi diable - alors que c'est un si grand homme et que je suis un moins que rien?»»30

Rassemblons les principaux éléments qui constituent le noyau de ce mythe: le caractère événementiel de la projection (non réitérable), dans le contexte d'une galerie (il est ici légitime de parler de «cinéma d'exposition"); l'interruption de la séance, conformément à la tradition surréaliste du scandale; la reconnaissance du caractère inaugural de ce cinéma de l'«objet trouvé» par Dali lui-même, qui accuse de façon très oulipienne Cornell de plagiat par anticipation; la stupeur et l'incompréhension qui font suite à la projection. Les souvenirs « reconstruits» de Levy (il publie ses mémoires en I977) accréditent l'œuvre de Cornell comme une alternative américaine au surréalisme - Cornell ne désirant en aucun cas être associé au mouvement - et plus précisément ses films comme une forme authentiquement «surréaliste» de cinéma. Des spécialistes du cinéma d'avant-garde tels que P. Adams Sitney ${ }^{31}$ ou Annette Michelson ${ }^{32}$ soulignent la dimension inaugurale du film de Cornell, malgré leur inscription dans des perspectives opposées: le film indépendant ou «visionnaire» pour Sitney, qui revendique la spécificité du champ du cinéma expérimental; l'art contemporain pour Michelson, qui ancre sa réflexion dans le structuralisme. De façon moins articulée et peu historicisée, ce film a pu être investi comme l'avènement du «postmodernisme» au sein même de l'apogée du «modernisme», hissant
30 Julien Levy, Memoir of An Art Galery, New York, Putman, 1977, pp. 23023I [ma traduction].

31 P. Adams Sitney, "The Cinematic Gaze of Joseph Cornell», dans Kynaston McShine (éd.), Joseph Cornell, New York, Museum of Modern Art, 1980, pp. 6889. Sitney, soulignant l'originalité du film, écrit notamment: «Rien de tel n'apparaîtra dans l'histoire du cinéma avant trente ans.»

32 Annette Michelson, «Rose Hobart and Monsieur Phot: Early Films from Utopia Parkway», Artforum, vol. II, $\mathrm{n}^{\circ}$ Io, juin 1973, pp. 47-57. 
33 «[... il répondait à l'art de son époque (le modernisme) d'une façon qui met en jeu l'art de notre époque (le postmodernisme). [...] Lorsque Cornell a commencé à faire de l'art, le collage était une technique bien établie que la plupart des artistes modernes mobilisaient [...]. Mais Cornell est l'un des premiers à investir exclusivement l'assemblage de matériaux trouvés comme médium et comme technique.» (Ingrid Schaffner, Joseph Cornell, New York, Harry N. Abrams/The Wonderland Press [The Essential Series], 2003, p. I4, p. I7).

34 Le colloque de Brighton consacré en r978 au cinéma des premiers temps (qu'on ne désigne dès lors plus comme "primitif») est généralement considéré comme le point de départ d'une «nouvelle» historiographie du cinéma. Voir par exemple Philippe Gauthier, «L'histoire amateur et l'histoire universitaire: paradigmes de l'historiographie du cinéma», Cinémas: revue d'études cinématographiques, vol. 2I, n 2-3 (Laurent Le Forestier [éd.], «Des procédures historiographiques en cinéma»), printemps 20II, pp. 87-105. Ce «tournant» historiographique pourrait cependant être lui-même interrogé et relativisé.
Cornell au rang de héraut de l'art du collage et de l'assemblage ${ }^{33}$, dans la filiation de Duchamp. Cependant, le relativisme critique lié à la «nouvelle» histoire du cinéma ${ }^{34}$ devrait nous inciter à revenir sur le contexte dans lequel s'inscrit ce film.

\section{La séance cinématographique comme forme d'exposition}

Joseph Cornell, tous les témoignages convergent sur ce point, est introduit à l'art d'avant-garde et au surréalisme en particulier par le biais de la Julien Levy Gallery, qu’il fréquente assidûment depuis son ouverture en I931. Julien Levy est l'un des premiers à importer le surréalisme aux Etats-Unis: en I932, il reprend, sous l'appellation sobre de

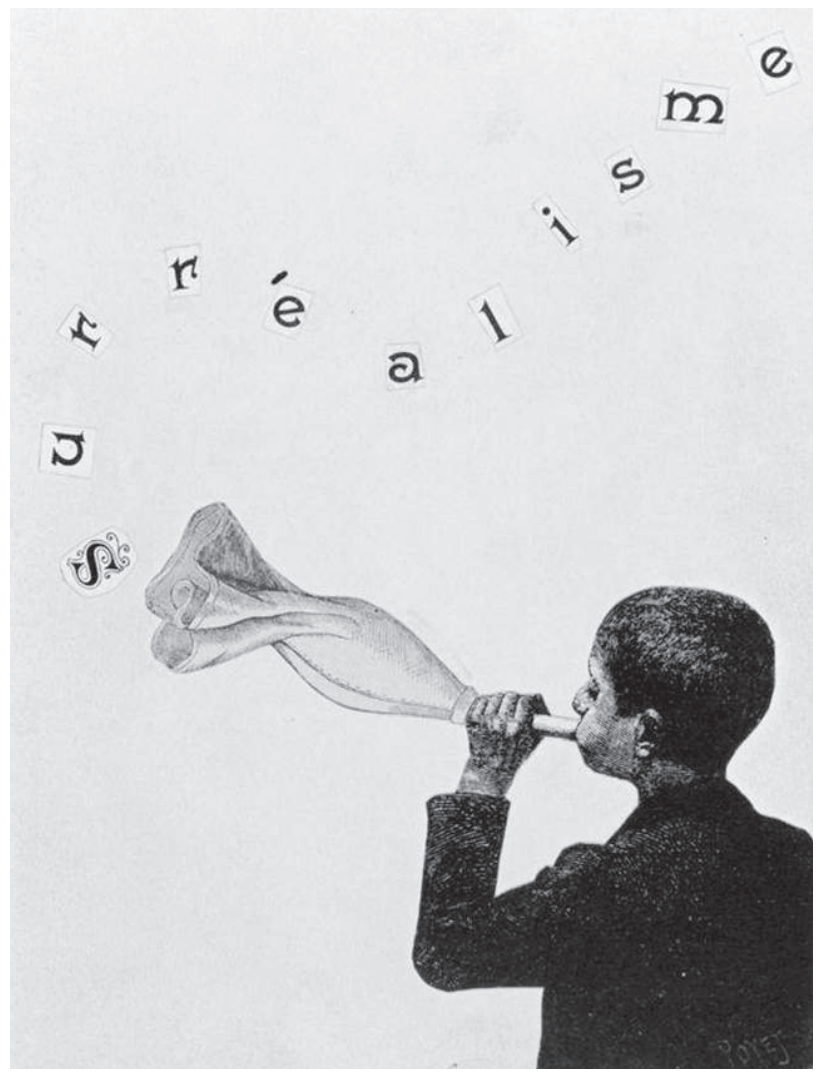


«Surréalisme», l'exposition «The New Super-Realism» organisée en novembre 193I par A. Everett Austin au Wadsworth Atheneum, à Hartford (avant que le MoMA n’organise d'événements en lien avec le surréalisme); dans ce contexte, il introduit de nouvelles œuvres, notamment américaines - Cornell figure ainsi dans l'exposition à travers des collages et des objets miniatures; et c'est à lui que Levy confie la réalisation de l'affiche, somme toute peu «surréaliste», de l'exposition: un enfant qui souffle à travers une trompette ou un tube de pâtissier les lettres «surréalisme». Auprès du rare public qui est attentif à ses activités artistiques, Cornell est définitivement associé à la Julien Levy Gallery: à l'occasion de sa première exposition «personnelle» (il est en fait exposé au côté de gravures de Picasso) en novembre i932, les objets de Cornell sont présentés par Levy comme des «jouets pour adultes» (l'expression, reprise dans les comptes rendus de presse, n'est pas des plus heureuses). Et sans aucun doute, son œuvre plastique - puis filmique - repose sur le collage et l'objet trouvé: elle assemble des objets et des signes disparates, dont la latence est révélée; elle exploite les potentialités du montage tout en renouant avec la pratique de la flânerie et du lèche-vitrine.

Cornell manifeste dans la plupart de ses boîtes et de ses dossiers une fascination pour des figures féminines, principalement des stars du cinéma classique telles que Hedy Lamarr, Lauren Bacall, Greta Garbo ou Jennifer Jones, qui sont discrètement érotisées ${ }^{35}$. Et, coïncidence qui n’a pas été assez soulignée de mon point de vue, le cinéma est également très présent dans les activités de Julien Levy: celui-ci organise chaque semaine des projections dans sa galerie; le film se trouve ainsi «exposé», au même titre que des collages, des assemblages ou n'importe quelle œuvre d'art. La séance de cinéma est ici investie comme l'équivalent d'une exposition collective; on assiste à l'émergence de la figure du programmateur en tant que curateur et en fin de compte en tant qu'auteur (ces termes sont anachroniques, mais désignent bien ce qui se noue déjà à cette occasion). L'implication de Julien Levy dans la culture cinéphilique qui émerge alors aux Etats-Unis - il fonde la New York Film Society avec Iris Barry, Raoul de Pussy de Salles et Nelson Rockefeller, notamment en vue de montrer L'Age d'or (I930) de Buñuel et Dali ${ }^{36}$ - peut être considérée comme l'embrayeur de l'engagement de Cornell dans la pratique filmique; sans aucun doute, elle détermine sa compréhension du cinéma. La séance de cinéma devient le lieu d’un
35 Sur ce point, voir l'étude bien documentée de Jodi Hauptman, Joseph Cornell: Stargazing in the Cinema, op. cit.

36 L'Age d'or (1930) est présenté en 1933 aux Etats-Unis (le film est censuré) à l'enseigne de la New York Film Society. Julien Levy avait inauguré ses séances hebdomadaires de cinéma dans sa galerie en I932 avec le programme suivant: Sportfilm (I932) de Victor Blum, Ballet mécanique (1924) de Fernand Léger et Anemic Cinema (1926) de Marcel Duchamp; il avait consacré sa deuxième séance au Chien andalou (1929) de Buñuel et Dali. Voir Julien Levy, op. cit., p. I55, pp. I49I5O. 
37 Voir le récit rétrospectif d'André Breton dans "Comme dans un bois", La Clef des champs, Paris, Pauvert, 1967 , pp. 290-296.

38 Sur ce point, voir François Albera, L'Avant-garde au cinéma, Paris, Armand Colin, 2005, pp. 7'-72.

39 Dans ses mémoires, Julien Levy affirme que la séance comporte un film de re-montage de Cornell, qu'il appelle Goofy Newsreel (Julien Levy, op. cit., p. 230). Dans la littérature secondaire qui porte sur Cornell, c'est la séance elle-même qui est baptisée Goofy Newsreel - dont le titre serait dû à Levy. Selon toute vraisemblance, Goofy Newsreel est bien un film de Cornell, alternativement intitulé Unreal Newsreels et édité sur le DVD Unseen Cinema - Early American Avant-Garde Film I894-I94I (New York, Anthology Film Archives, 2005).

40 Julien Levy, op. cit., p. 230 [ma traduction]. montage de films préexistants, s'apparentant à une collection d'objets donnés à voir à travers un point de vue curatorial orienté. L'acte de création, suivant la logique de l'appropriation, dépend ici étroitement de l'activité du programmateur - et du spectateur, comme l'avaient découvert les surréalistes, André Breton et Jacques Vaché prélevant des séquences de films en entrant par hasard dans des salles de cinéma ${ }^{37}$. Les films de re-montage de Cornell participent de plain-pied à un regard surréaliste sur le cinéma et en fin de compte à un «cinéma de spectateur» ${ }^{38}$ : Cornell prélève des films ou des fragments de films qui s’inscrivent dans la culture populaire, et les re-monte ou les donne à voir à travers un geste de «désautomatisation» de la perception ou de «défamiliarisation» de l'objet.

C'est ici le lieu de rappeler que Rose Hobart était projeté avec d'autres films d'avant-garde, formant une séance composite: en l'occurrence Anemic Cinema (1926, Marcel Duchamp), L'Etoile de mer (1928, Man Ray, d'après Robert Desnos) et Goofy Newsreel (circa 1926-1928, Joseph Cornell) ${ }^{39}$. Levy soutient que cette séance tend à «illustrer les inversions du mot et de l'image - une imagerie muette ou un langage imaginaire ${ }^{40}$. Ce qui paraît indéniable, c'est qu'à cette occasion Cornell adhère à la logique du programmateur de films: la séance devient elle-même le lieu
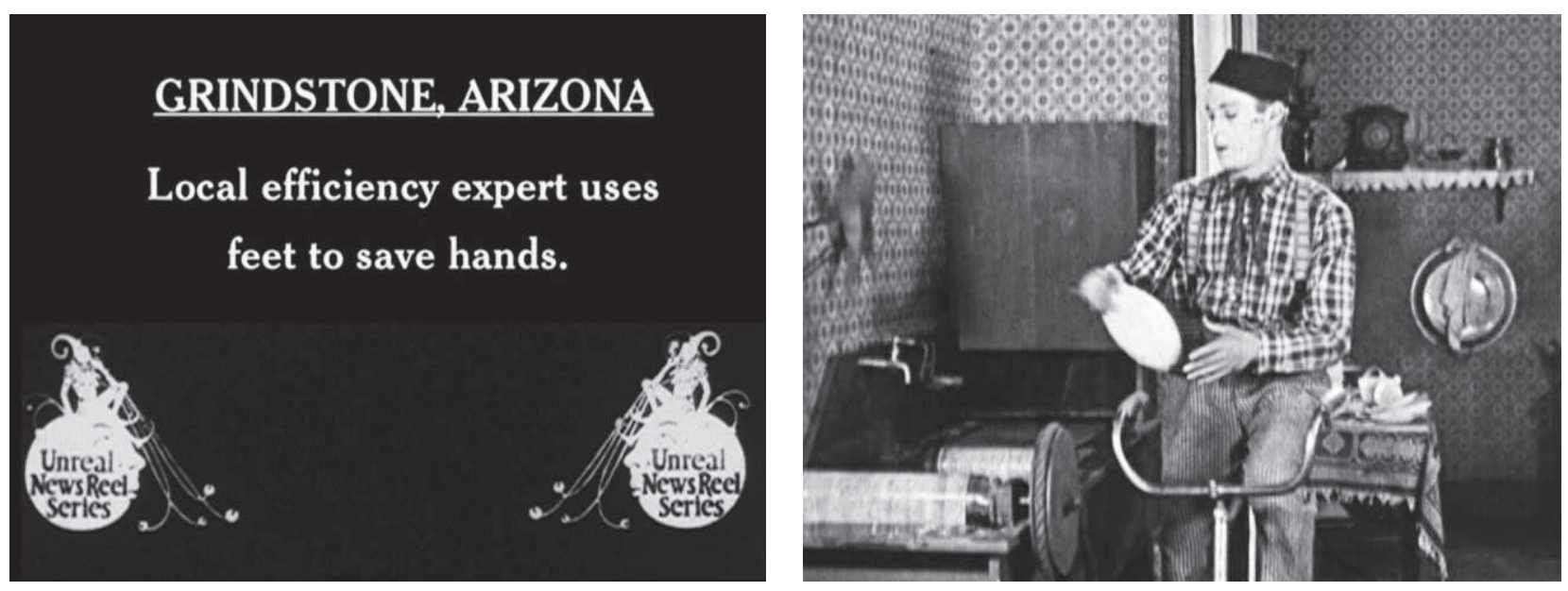
d'un re-montage de films d'avant-garde, formant un ensemble ouvert. En ce sens, la séance revêt un aspect performatif ${ }^{41}$ : c'est dans l'acte de son déroulement que le re-montage s'actualise, le projectionniste - Cornell en personne - acquérant le statut d'auteur ou de co-créateur.

Rose Hobart, ainsi contextualisé, s'émancipe du dispositif de la salle obscure, qui est déterminé par le primat d’une vision centrée: la logique qui prévaut est celle de la distraction et du dérèglement de la vision. Comme le souligne Ken Jacobs, le film devient d'autant plus troublant s'il est diffusé sur une autre surface que l'écran, à travers une vision de guingois (Ken Jacobs, avec Jack Smith, le projetant par la suite sur le plafond ou un coin de mur) ${ }^{42}$. Levy met en place un cadre singulier de monstration: il réinscrit l'expérience de la séance cinématographique au sein de l'espace économique et idéologique de la galerie (le white cube ${ }^{43}$ ). Ce dispositif hybride ouvre les conditions de possibilité d'un dépassement des conventions du film et des contraintes de l'exposition: Cornell réinvente un «cinéma de spectateur», englobant l'ensemble des films présentés (dont ses propres réalisations) dans une forme de séance «surréaliste», à travers la dynamique d'un work in progress. Ce geste d'appropriation et de réélaboration seconde du cinéma est mis en abîme à travers Goofy Newsreel: ces «actualités du rêve» ou de l'«irréel» reposent sur un geste de décontextualisation et de recontextualisation de plans, en mobilisant l'horizon d'attente des actualités filmées (des séquences de films comiques - avec Keaton, Arbuckle, des imitateurs de Charlot, etc., de nombreux plans étant empruntés aux productions des Weiss Brothers Artclass Pictures - sont précédées d’intertitres comportant le nom d'un lieu et l'énoncé d'une «nouvelle» fantaisiste). Un film burlesque acquiert ici le même statut qu'une vue d'actualité, et inversement. L'intertitre assigne un sens - des plus improbables - au plan: ce cinéma fort singulier et anonyme se voit contradictoirement dés-autorisé (évoquant par anticipation la «mort de l'auteur») et ré-auratisé (révélant la latence des plans).

La séance cinématographique en galerie telle que la conçoit Levy et Rose Hobart de Cornell - la séance «surréaliste» se trouvant pour ainsi dire condensée dans l'espace d'un seul film - ne sont pas incompatibles avec le projet d'une «métahistoire du cinéma» telle que la définit Hollis Frampton ${ }^{44}$, au centre de son dernier cycle épique et inachevable (Magellan, 1972-1980). Dans tous les cas, il s'agit rien de moins que de
41 Sur la dimension performative du cinéma de Cornell, voir Erik Bullot, Sortir du cinéma, op. cit., pp. I4I-I63.

42 Ken Jacobs, alors qu'il travaillait comme assistant pour Joseph Cornell, lui a emprunté une copie de Rose Hobart. Avec Jack Smith, il projette ce film «de toutes les façons possibles: sur le plafond, sur des miroirs, se réfractant à travers la chambre, sur des recoins, en faisant ou non le point, à travers un filtre bleu donné par Cornell, sans filtre, en arrière...» (entretien de Ken Jacobs par P. Adams Sitney, cité dans P. A. Sitney, Visionary Film: The American AvantGarde, op. cit., p. 349).

43 A cet égard, voir la critique radicale de Brian O’Doherty, White Cube - L'espace de la galerie et son idéologie, Zurich, JRPIRingier, 2008 [première publication: Inside the White Cube. The Ideology of the Gallery Space, Santa Monica/San Francisco, The Lapis Press, I986].

44 Voir Hollis Frampton, «Pour une métahistoire du film: notes et hypothèses à partir d'un lieu commun", L'Ecliptique du savoir [Annette Michelson et Jean-Michel Bouhours (éd.)], Paris, Centre Georges Pompidou, I999, Pp. I03-III [première publication: «For a Metahistory of Film: Commonplace Notes and Hypotheses", Artforum, vol. Io, nº I, septembre i97I, pp. 32-35]. 
45 Comme il l'exprime clairement dans une demande de bourse à la Fondation Guggenheim, le projet de Frampton n'est pas de rassembler ou re-monter l'histoire du cinéma à partir de films préexistants, mais de réaliser des films qui suppléent l'histoire du cinéma qu'il juge inaboutie, voire déficiente: l'enjeu est de «refaire le cinéma comme il devrait être» (Hollis Frampton, «Proposition» [circa I972], cité dans H. Frampton, L'Ecliptique du savoir, op. cit., pp. I74-I75).

46 Walter Benjamin, Paris, capitale du $\mathrm{XIX}^{e}$ siècle. Le livre des passages, Paris, Cerf, i989, p. 224.

47 Id., p. 222.

48 Id., p. 224. réécrire l'histoire du cinéma en inventant une nouvelle tradition. Mais chez Cornell, à la différence de Frampton ${ }^{45}$, le point de vue du collectionneur se superpose à celui du «métahistorien» du cinéma.

\section{Le fétichisme du collectionneur}

Cornell, on le sait, est un collectionneur: il a accumulé un large ensemble de photographies de danseuses, de divas d'opéra et de stars de cinéma (en acquérant notamment des images publicitaires destinées aux exploitants de salle), parmi d'autres figures féminines; et il a constitué une importante collection de films des premiers temps et des années I920, notamment des films à trucs, des films d'animation, des films de voyage, des documentaires animaliers et éducatifs, des films burlesques et des «classiques» des années I920-I930 (il lègue 150 bobines à Anthology Film Archives en I97I). Sa pratique filmique gagne à être considérée dans le prolongement de cette compulsion à acquérir et à «cataloguer» des films. Les réflexions de Walter Benjamin sur la figure du collectionneur permettent d'éclairer le positionnement de Cornell dans le champ du cinéma, rectifiant en quelque sorte la connotation d'«écriture automatique» attachée à l'«objet trouvé» que j’ai d'abord mobilisée (et qui nécessite pourtant de longues recherches, quand bien même celles-ci seraient-elles orientées par le «hasard objectif»).

Benjamin soutient que «le vrai collectionneur détache l'objet de ses rapports fonctionnels ${ }^{46}$, entretenant avec celui-ci une relation qui n'est pas sans évoquer la «contemplation désintéressée» dans l'esthétique du Beau de Kant (ce point de vue est caractéristique d'une sensibilité surréaliste). La valeur d'usage des objets s'estompe face à leur appréhension en tant que monde clos, chaque chose pouvant «nouer la relation la plus étroite possible avec les objets qui lui sont semblables» ${ }^{47}$. De plus, la constitution de l'objet en une «collection» permet de l'intégrer à un système signifiant nouveau, établissant une relation inédite de l'objet au monde et des objets entre eux:

«[...] pour le collectionneur, le monde est présent et, qui plus est, rangé dans chacun des objets qu'il possède. Mais rangé selon un agencement surprenant et même incompréhensible au profane. Cet agencement est à l'organisation et à la schématisation ordinaires des choses ce que leur ordre dans une encyclopédie est à un ordre naturel.» ${ }^{48}$ 
On ne saurait mieux décrire la logique d'agencement des plans dans les films de re-montage de Cornell. En effet, chaque plan suit un système de relations complexe et surprenant: la fonctionnalité des images est ainsi désamorcée, la référentialité première du film suspendue; formant un monde clos, les plans tissent des liens entre eux qui ne sont pas motivés par la raison ou le récit, mais par des rapprochements formels ou rythmiques, par des réitérations et des déclinaisons, par des oppositions thématiques ou iconographiques. Seul le re-monteur maîtrise les lois et la logique de ce système «encyclopédique».

Benjamin ne manque pas de souligner la pensée magique qui oriente le collectionneur de livres (s'incluant lui-même dans ce portrait), dont la visée n'est pas seulement encyclopédique (on retrouve ici la «magie blanche» chère à Cornell):

"Chez les enfants, l’acte de collectionner n'est qu’un procédé de renouvellement parmi d'autres, tels la peinture des objets, ou le découpage, ou encore le décalque [...]. Renouveler le monde - c'est là l'instinct le plus profond dans le désir qu'éprouve le collectionneur d'acquérir de nouveaux objets [...].» ${ }^{49}$

Lacte de collectionner des films et des images, s'inscrivant dans le prolongement naturel du collage, du découpage et de l'assemblage d'objets, s'apparente chez Cornell à une éthique du renouvellement: les objets sont défamiliarisés, et notre perception à leur égard renouvelée. Collectionner, c'est déjà réassembler les objets en un système signifiant inédit: c'est déjà «re-monter». Le geste d'agencement du collectionneur chez Cornell, lorsqu'il transite par le support du film ${ }^{50}$, est le plus souvent orienté par les modèles de la fantasmagorie, de la féerie et des vues à transformation.

\section{Le regard ébloui de l'enfant, ou l'attraction des vues à transformation et la généralisation du faux raccord}

Cornell a développé un vaste ensemble de films de collage à partir de sa collection privée (acquise auprès de vendeurs et au hasard de ses pérégrinations dans divers marchés aux puces), sans qu'il soit toujours possible de déterminer si le film est complet ou sa finition indéfiniment différée. Débutés dans les années I930, ceux-ci seront parfois terminés dans les années I970 par Larry Jordan, suivant les instructions de Cornell. Ces films qui empruntent des plans à des sources multiples, la plupart du
49 Walter Benjamin, Je déballe ma bibliothèque [1931], Paris, Payot (coll. Rivages), 2000, p. 44 .

50 Cornell peut également re-disposer les éléments du dispositif cinématographique (mais aussi d'autres formes spectaculaires: le ballet, l'opéra) sous forme de boîtes et de dossiers destinés à l'exposition. C'est le cas de The Romantic Museum: Portraits of Women, exposé à la Hugo Gallery en 1946. Sur ce point, voir Jodi Hauptman, op. cit., pp. II-2I. 
51 Children's Trilogy comporte les films suivants: Cotillion (circa I938), The Midnight Party (circa 1938) et The Children's Party (circa 1938).

52 Marjorie Keller, The Untutored Eye: Childhood in the Films of Cocteau, Cornell, and Brakhage, Rutherford/Londres/ Toronto, Fairleigh Dickinson University Press / Associated University Presses, I986, p. I40, p. I43 [ma traduction]. temps difficilement identifiables, manifestent une obsession pour le contexte forain, en particulier le cirque et le zoo; les scènes de vaudeville, les spectacles de magie, les tours de cirque s'enchaînent sous le regard ébahi d'enfants, relais du point de vue du spectateur. Plutôt qu'à des stars (féminines) de cinéma, Cornell rend ici hommage à la dynamique «attractionnelle» du cinéma des premiers temps (Cornell éprouvant également une fascination pour Lumière, Méliès, Zecca, ou encore Cohl). L'illusionnisme visuel et les trucages «primitifs» rivalisent avec la logique du trompe-l'œil et un montage dont la logique procède à première vue du coq-à-l'âne (en fait, il n'en est rien: l'assemblage des plans est rigoureux). Marjorie Keller note avec pertinence la présence de jeux de mots qui structurent certains de ces films; elle écrit ainsi, à propos du dernier film de The Children's Trilogy ${ }^{51}$ :

«Children's Party est centré sur le thème des étoiles [stars]: les étoiles dans le ciel [stars in the sky], les enfants étoiles [child stars], les étoiles de cirque [circus stars] et la lumière des étoiles [starlight]. [...] Cornell, à l'instar de Cocteau, transpose littéralement la relation entre les étoiles dans le ciel et les étoiles sur scène ou dans un film [stage and movie stars]. Si l'on se réfère à d'autres œuvres de Cornell, il paraît évident qu'il a élaboré des jeux de mots visuels à partir de constructions verbales: en l'occurrence, ébloui par les étoiles [star-struck], des étoiles dans les yeux [stars in his eye] et foudroyé par le sort [star-crossed].» ${ }^{52}$

Dans cette trilogie, où les mêmes matériaux filmiques sont remontés, le fétichisme de Cornell est redirigé sur de jeunes filles, qui sont à la fois le sujet et l'objet du regard. Comme le souligne Marjorie Keller, les séquences d'attraction (une acrobate suspendue à un fil, une funambule, une jeune écuyère) et les phénomènes astraux (des télescopes, la voie lactée, Zeus qui lance des éclairs) sont assemblées par rapport à une logique verbale sous-jacente, difficilement perceptible, qui procède par glissements lexicaux, substitution d'une expression lexicalisée par une autre métaphore figée. Néanmoins, il ne me paraît pas possible de rapporter les différents films de collage de Cornell à un procédé commun, à une stratégie de structuration unique. J'identifierai tout au plus au sein de cet ensemble deux pôles en tension, deux paradigmes opposés: la dislocation des plans à travers une pratique généralisée du faux raccord d'une part, la métamorphose incessante des vues à travers différents trucages d'autre part. 
The Children's Party (circa I938) repose sur une poétique de l'interruption généralisée: lorsqu’une logique sérielle tend à se constituer, elle est aussitôt perturbée par un ordre divergent, quitte à refaire surface par la suite - avant une nouvelle bifurcation ou la reprise d'un précédent embranchement. Le film repose sur trois principaux vecteurs thématiques ou séries iconographiques: la musique (ou les musiciens), le véhicule (ou le déplacement), le primitivisme (ou l'archaïsme de la nature et des Amérindiens). Le passage de l'un à l'autre de ces ensembles se fait par courtcircuit, désamorçage, dislocation ou disruption. Cette discontinuité est soulignée par la bande-son, constituée d'un pot-pourri de chansons et d'airs musicaux en vogue, de bruits de la jungle et de dialogues interrompus (il serait légitime de parler de musique concrète en ce cas).

Le film s'ouvre sur deux explorateurs accrochés à une branche d'arbre, qui amorcent un récit de voyage stéréotypé aussitôt interrompu; le plan suivant cadre deux bûcherons qui entreprennent de scier le tronc d'un énorme arbre. Les espaces sont disjoints, mais une logique d'inversion - de chute - est discrètement suggérée. Coupe sèche: une voiture de pompier sort d'une caserne; autre plan, elle traverse une rue. L'éventualité d'un feu permet de relier ces deux séquences - le bois brûle, et la voiture de pompier n'est pas tenue de respecter les feux de circulation mais la rapidité de l'enchaînement procure une impression de montage décousu, aléatoire. Suit un plan sur un pianiste (qui affirme être né avec une voix en or): soit l'introduction de la série musicale. Retour à l'espace primitif de la forêt: un éléphant est cadré au premier plan; puis, un tracteur tire un tronc d'arbre (répondant ainsi à l'action de scier un arbre); vue saugrenue, deux personnages se tiennent debout, en équilibre, sur le tronc; à nouveau, on voit à travers un autre angle de prise de vue le tracteur qui tire un tronc (sans personne dessus cette fois); un nouveau cadrage donne l'impression que le tracteur monte une pente abrupte; enfin, des éléphants tirent un tronc d’arbre. A travers une série de faux raccords, la modernité du tracteur et le «primitivisme» des éléphants tendent à s'intervertir. Une variante sur le thème de l'éléphant est proposée (des éléphants de cirque, qui laissent place à des clowns qui se griment), avant de revenir au thème du pianiste (une chanteuse est cadrée à l'envers; le pianiste est filmé à l'envers; puis il est recadré normalement). Retour aux véhicules, avec une nouvelle variation: un train, avec des voyageurs qui montent; le véhicule se met en branle; etc. Les faux 
raccords introduisent des éléments qui semblent répondre à une logique de l'équivalence généralisée; mais Cornell rompt avec ce rythme syncopé et ces séries: une école est présentée; une jeune fille trie du coton; un avion effectue une série de vols acrobatiques: le pilote embrasse une jeune fille; à cette vue se substitue la photographie d'un couple royal; retour sur le pilote et la jeune femme; nouvelle rupture ou embranche-

Children's Party (Joseph Cornell, circa 1938)
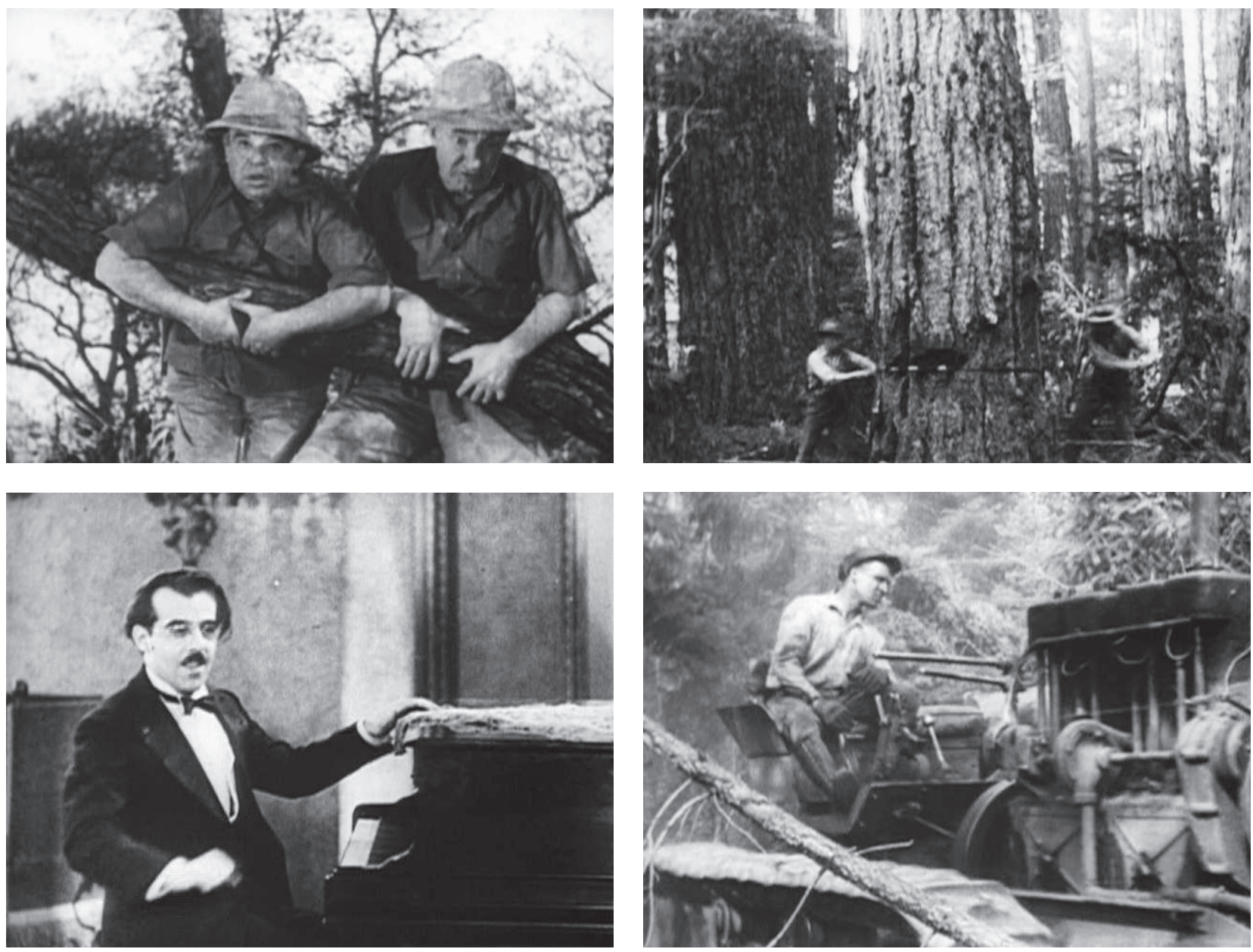
ment, des Amérindiens s'adonnent à une danse rituelle. La séquence de danse rituelle, plutôt longue, avec des changements d'angles de prises de vues, traduit à un niveau manifeste la logique latente du film: c'est-à-dire l'apologie d'un monde d'avant la civilisation, suivant un fantasme primitiviste caractéristique de la pensée de Freud (je pense à son essai «La morale sexuelle 〈civilisée〉 et la maladie nerveuse des temps modernes ${ }^{53}$
53 Sigmund Freud, «La morale sexuelle 〈civilisée` et la maladie nerveuse des temps modernes», La Vie sexuelle, Paris, PUF, 1969, pp. 28-46 [première publication: «Die <kulturelle`, Sexualmoral und die moderne Nervosität», Studienausgabe, vol. 9, 1908].
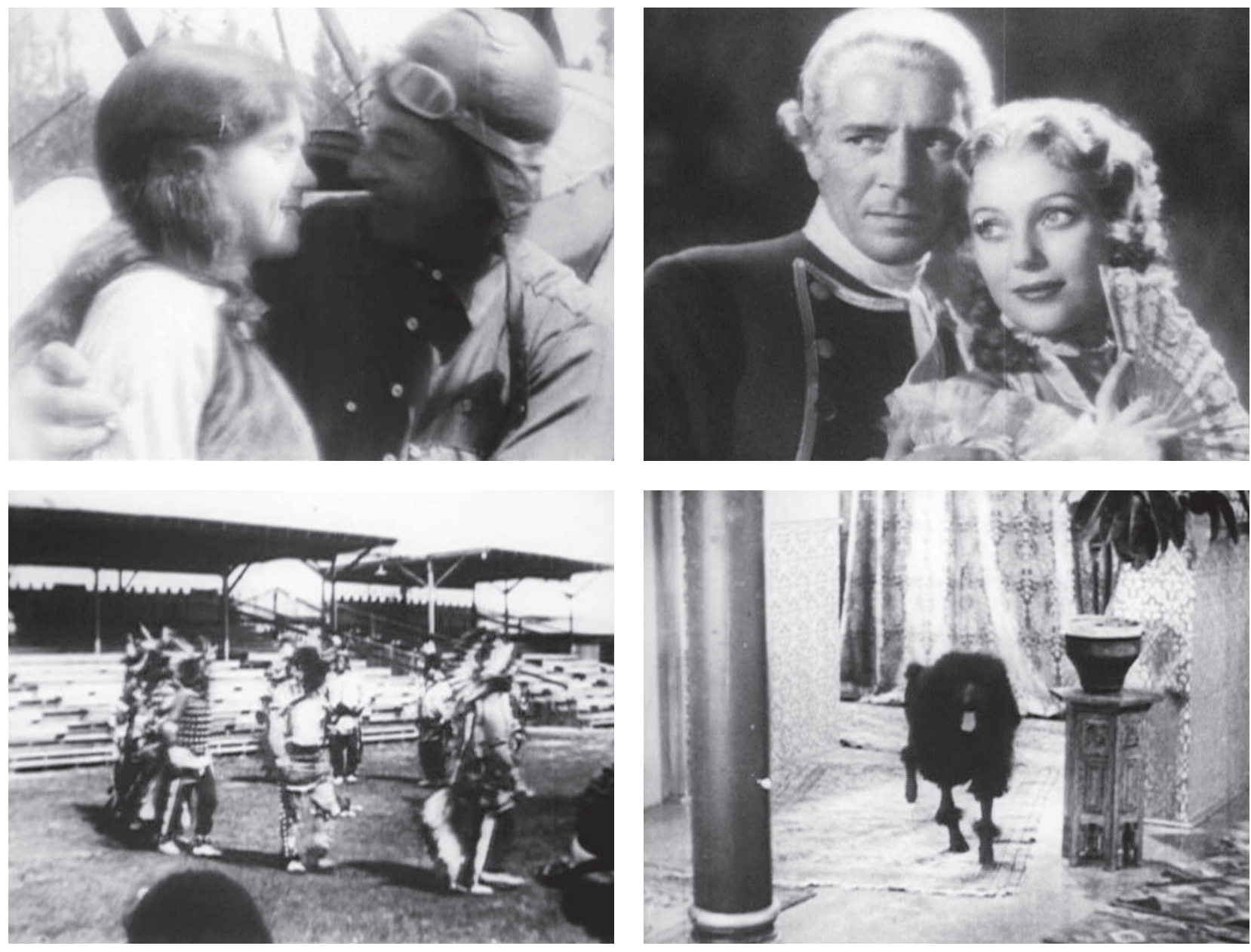
54 Sigmund Freud, Le Malaise dans la culture, Paris, Payot, I967 [première publication: Das Unbehagen in der Kultur, Vienne, Internationaler Psychoanalytischer Verlag, I930]. et à son ouvrage Malaise dans la culture $\left.{ }^{54}\right)$. Je suspends là la description du film - qui se clôt sur un chien qui court dans une luxueuse propriété, sur le visage d'un enfant noir qui pleure, avant la mention «THE END» dont les caractères sont inversés.

Dans une perspective diamétralement opposée, Thimble Theater (circa I938, achevé en I970 par Jordan) remet en jeu la dynamique des
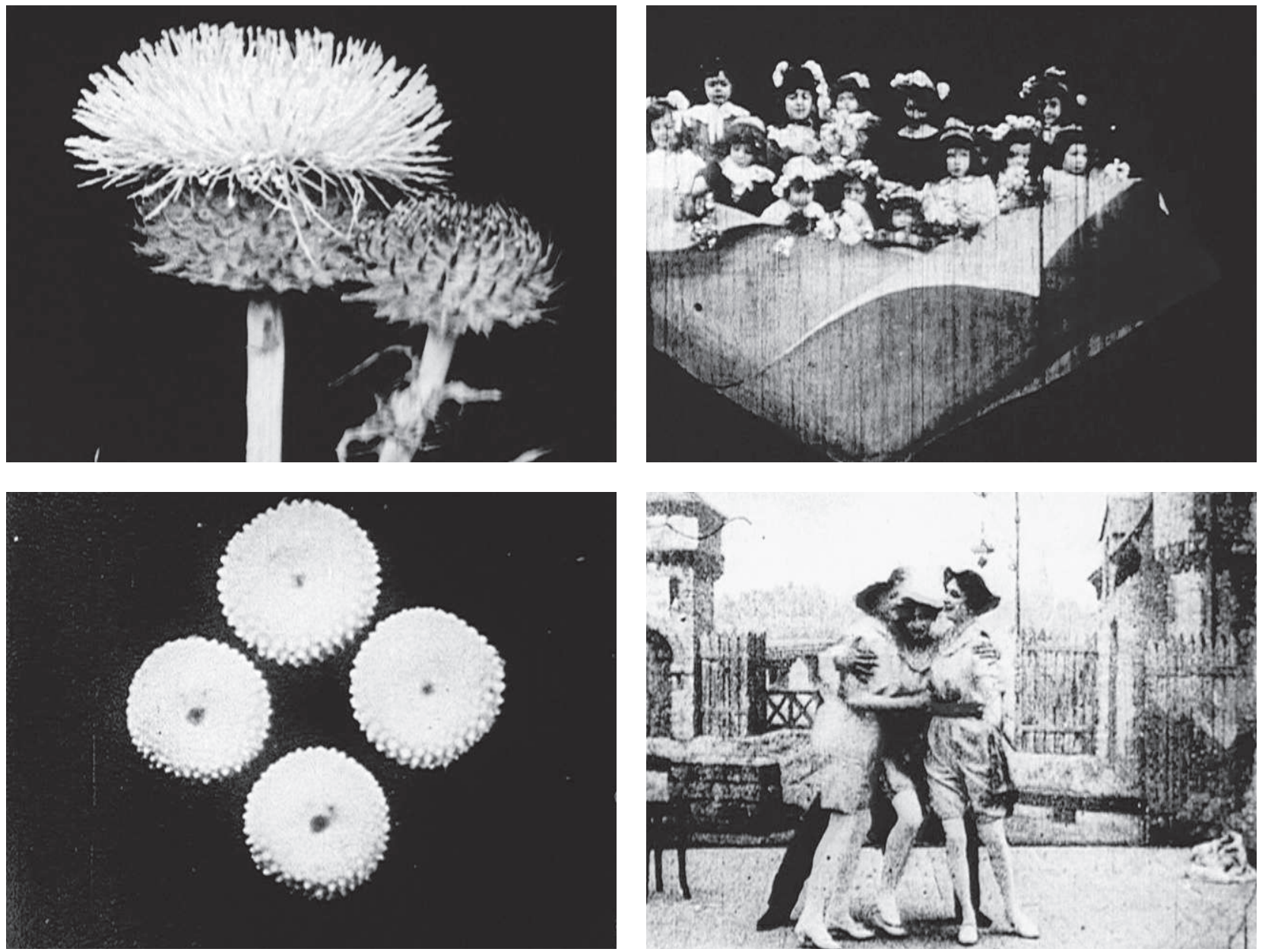
vues à transformation, misant sur la continuité entre les scènes et un théâtre de l'anamorphose généralisée - la bande-son est composée d'une mélodie continue de type carrousel. Le prologue donne le ton: des corolles de fleur, en gros plans, s'ouvrent (deux plans); des lions sautent à travers un cerceau enflammé; des pélicans voguent sur l'eau; des jeunes filles, dans un décor de fleur en papier géante, regardent un spectacle que
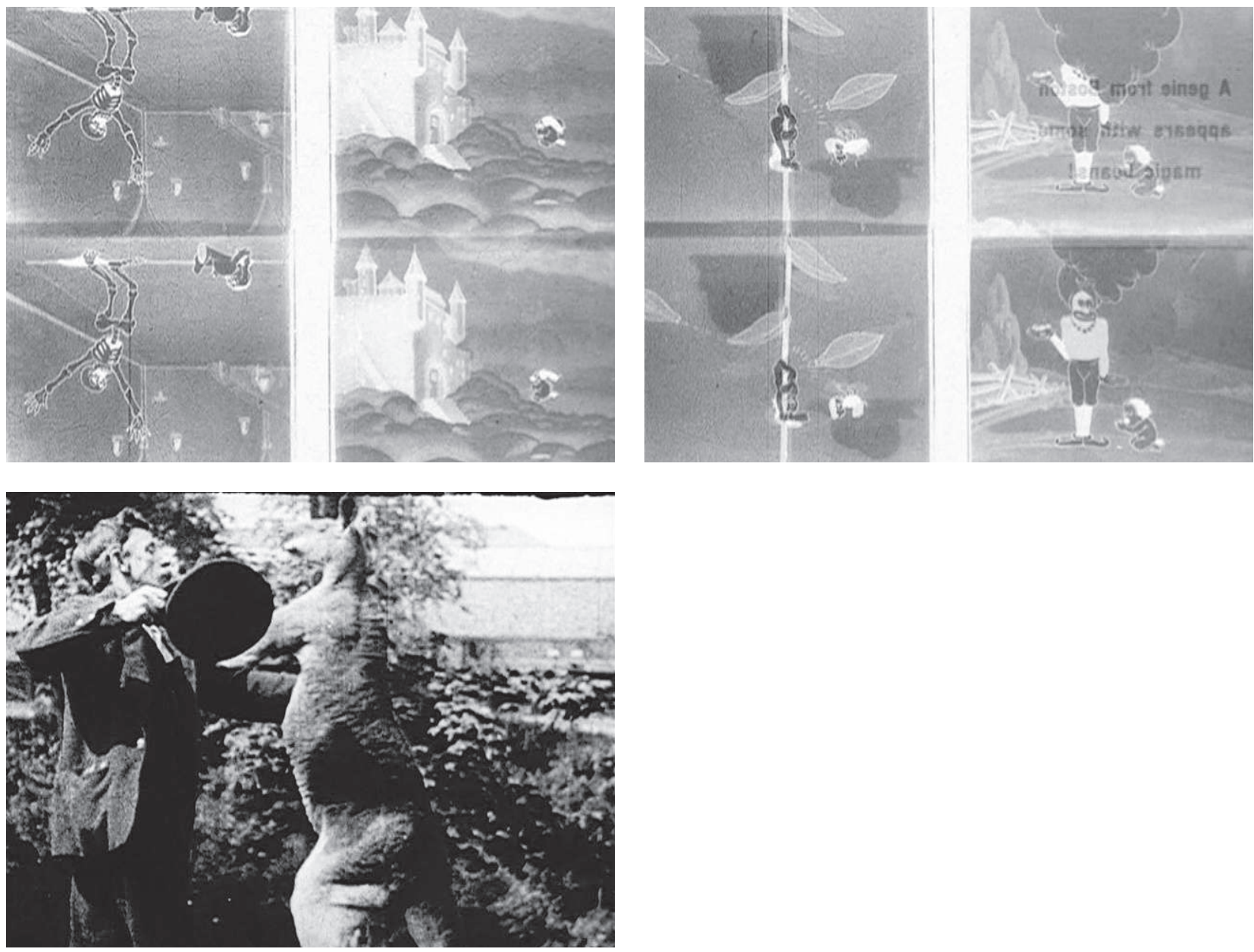
le spectateur ne peut voir. La rotondité permet d'enchaîner les premières séries de plans, tandis que la métaphore florale fait le lien avec les jeunes filles, suivant une logique d'association verbale. Une série d'amorces abstraites, qui font suite au décompte du pré-générique, précèdent la mention: "A film from the collection of Daniel Luna». L'attribution de ce film de re-montage à un personnage au nom lunaire est caractéristique de la poétique de Cornell. Les motifs floraux sont ensuite déclinés dans les plans suivants, avant de laisser place à des matériaux hétéroclites, donnés à voir à travers une certaine durée. Un film scientifique sur la transformation des chenilles en papillons (un intertitre identifie le statut documentaire de cette source: «Nature's Handiworks: Wondrous phases of life and transformation of caterpillars moth-butterflies») donne le ton; la métamorphose est le principe de construction de Timble Theater. Un film à trucs de Méliès prolonge et redirige cette logique anamorphique: un homme fait apparaître des femmes, leurs corps se substituant au sien; le transformisme redessine ce passage des corolles de fleur aux jeunes filles. Suit un dessin animé dédoublé et dupliqué en négatif, la partie occupant la gauche de l'écran défilant à l'envers avec un léger décalage temporel: le passage au négatif "primitivise» cette fantaisie autour de Jack et le haricot magique. Une série animale complète cette logique de dérivation: des chamois se déplacent le long de rochers, vraisemblablement dans un zoo; apparaissent ensuite des kangourous, avec des plans repris au ralenti. La conjonction homme-animal est ainsi actualisée à travers une séquence «burlesque» où le gardien du zoo boxe avec l'un des kangourous. L'attraction ici est relative à la nature des séquences montées et au point de vue que Cornell porte sur celles-ci, relayé à plusieurs reprises par le regard de jeunes filles: l'éblouissement est mis en scène dans les plans cités et exacerbé par le re-montage. La conjonction entre avant-gardisme, attraction du cinéma des premiers temps et primitivisme est ici sciemment exploitée.

Ces films de re-montage - qui sont parfois d'une simplicité désarmante: dans Carrousel - Animal Opera (circa 1938), Cornell monte bout à bout différents animaux dans un zoo, proposant un ballet ou une chorégraphie d'attitudes anthropomorphisées à partir de raccords de continuité, établissant par exemple un lien entre le bâillement d'un hippopotame et le rugissement d'un lion - représentent un équivalent cinématographique de la stratégie duchampienne du ready-made rectifié ou 
assisté. Mais chez Cornell, il suffit parfois de l'adjonction d'un seul plan pour que le film décroche ou décolle dans une autre dimension. Ce sera là mon dernier exemple: dans Jack's Dream (circa 1938, achevé par Jordan en I970), le monde clos, autonome, d'une animation de poupées, est soudainement fissuré par l'introduction de plans figuratifs, photographiques. Le film s'ouvre sur un livre, comme pour signifier son irréalité:

Jack's Dream (Joseph Cornell, circa 1938)
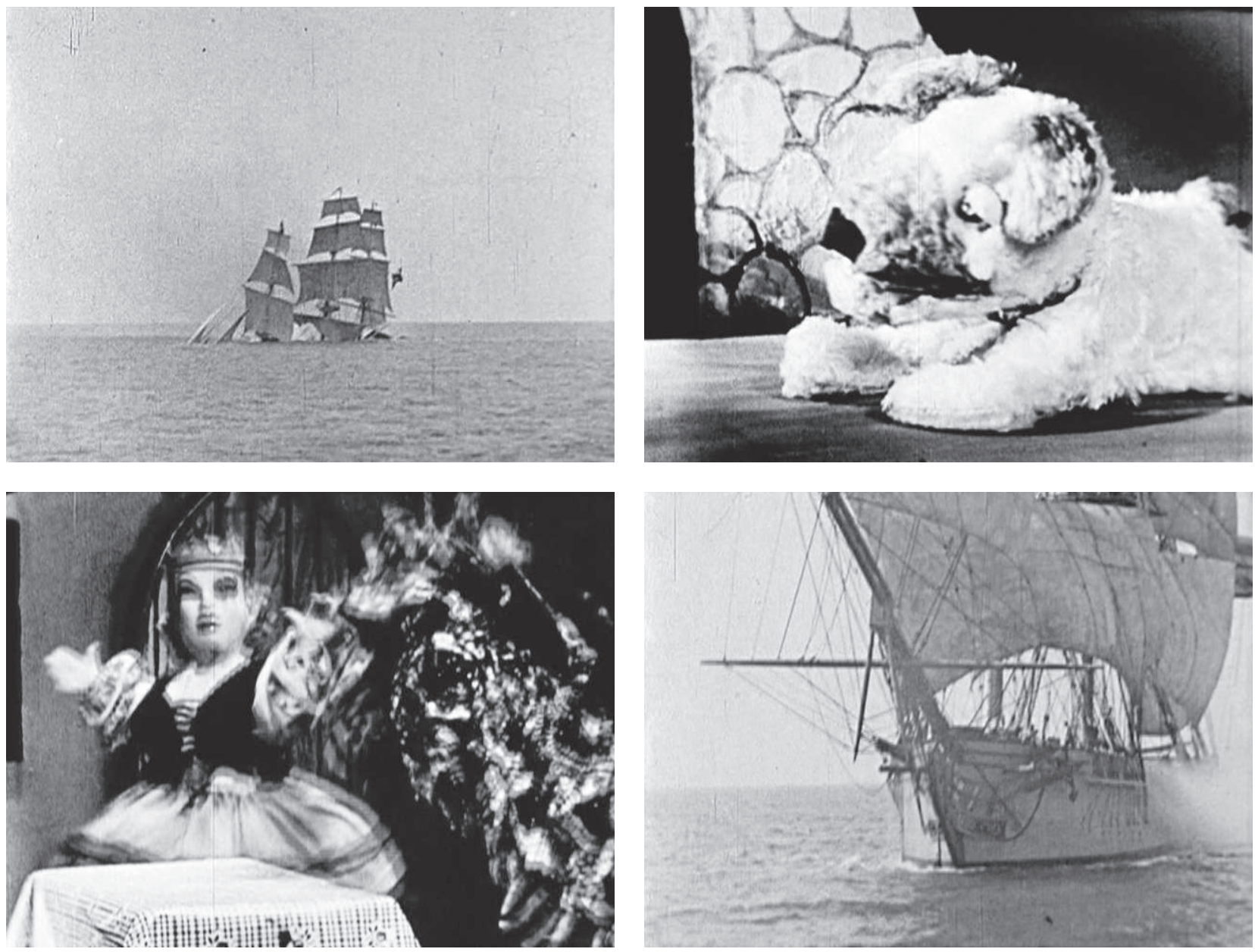
55 Voir Maurice Blanchot, L'Ecriture du désastre, Paris, Gallimard, 1980.
56 Voir la biographie de Cornell: Deborah Solomon, Utopia Parkway: The Life and Work of Joseph Cornell, New York, Farrar, Straus and Giroux, I997. il s'agit du Petit Chaperon rouge; passé la page de titre, une illustration s'anime: à travers une fermeture à l'iris, le spectateur pénètre à l'occasion du plan suivant dans l'univers féerique de l'animation. Cornell multiplie les déplacements par rapport au conte du chaperon rouge: le loup est remplacé par un dragon, le cadre de l'action est celui d'un château, tandis qu'un chien de compagnie se révèle omniprésent. Mais le soudain basculement du film dans l'attraction ne repose pas sur ces licences prises à l'égard du récit cadre. A l'opposé de la logique d'ancrage dans un mode de référentialité direct qui caractérise l’irruption de l'image photographique «conventionnelle» ou «plate» dans le domaine de l'animation, c'est l'inverse qui se produit: le plan «documentaire» plonge le film dans le domaine de la fantasmagorie, et ceci à deux reprises. Des personnages lavent la vaisselle dans le château; l'âtre de la cheminée crépite; le chien est couché à côté du feu; soudainement, un navire disparaît sous les flots; des hippocampes se déplacent sous l'eau (on pourrait y voir une réminiscence de certains films de Painlevé, qui est lui aussi associé au surréalisme). Le plan du naufrage du navire fracture l'unité diégétique et formelle du film, qui bascule dès lors dans une poétique du «désastre» généralisé (du Coup de dés de Mallarmé aux relectures de Blanchot, qui comprendra ce terme dans son sens étymologique: «chute de l'astre» ${ }^{55}$ ). Seconde occurrence: le dragon, menaçant, s'attaque aux êtres humains; brusquement, un bateau tire des coups de canon; le chien s'endort; suit une vue du ciel, probablement depuis le bateau. Au niveau du déroulement logique, ce plan «figuratif» précède le naufrage du navire, en en présentant la cause; mais il clôture ici la narration du film à travers un geste de défense (le bateau se substituant au chasseur du conte) - qu'il faut peut-être attribuer au rêve du chien. La dimension documentaire de la photographie, à travers un étonnant coup de force, devient ici un vecteur d'irréalité et de «surréalité». L'univers de Cornell repose sur une série de basculements, la poétique de l'«infra-mince» qu'il privilégie (conformément à la vie banale de l'artiste, résidant chez sa mère dans un morne pavillon de banlieue - toutefois sis à l'adresse Utopia Parkway ${ }^{56}$ - et tenant compagnie à son frère handicapé) constituant très vraisemblablement une ultime inversion des faits hauts en couleurs des surréalistes.

Les bricolages filmiques de Cornell déplacent en fin de compte la logique du ready-made aidé: si Duchamp décontextualise et renomme 
un objet usuel manufacturé (par exemple un porte-bouteilles, une pelle à neige ou encore un urinoir) en l'assignant à «l'institution art» ${ }^{57}$, Cornell accole une nouvelle légende à des plans ou des fragments de films rendus à leur anonymat et investis en tant qu'«instants prégnants» ${ }^{58}$. Cornell, avec l'aide et le soutien de Levy, substitue une aire de jeu indéterminée à l'espace économique et idéologique de la galerie, en souscrivant à la logique du don (qui engage une réciprocité de la part de son destinataire: le «contre-don», d'après la définition de Marcel Mauss ${ }^{59}$ ). La séance cinématographique de Cornell - ou faudrait-il écrire: de Levy? - propose une série de «variations» (dans le sens musical du terme) à partir de plans préexistants, qui sont réactualisés par le biais de nouvelles séquences entretenant avec ceux-ci une relation dynamique (qui peut être spécifiée, en première approximation, comme une opération de déplacement spatial et temporel, de requalification et de resémentisation, suivant un processus d'appropriation énonciative). Le re-montage est ainsi démultiplié à travers une vertigineuse mise en abyme (dont la logique est fort distincte de la mise en relation thématique ou par contiguïté spatiale de vues par un exploitant de salle dans les premiers temps du cinéma ${ }^{60}$ ): en premier lieu, les films de compilation de Cornell re-montent des plans hétéroclites (s’il fallait citer des antécédents, je renverrai aux films d'Adrian Brunel qui réagencent des vues d'actualités en leur adjoignant des cartons fantaisistes ${ }^{61}$ ); en deuxième lieu, la séance de Cornell réarticule les films et les plans en un ensemble inédit, superposant différentes temporalités et gestes intentionnels en un nouveau continuum; et, en dernier lieu, c'est au spectateur qu'incombe la responsabilité finale du re-montage, l'intention signifiante de la séance pouvant connaître un ultime déplacement (voir la lecture paranoïaque de Dali du film de remontage de Cornell, Rose Hobart).
57 Sur la logique du ready-made, voir Thierry de Duve, Résonances du readymade: Duchamp entre avant-garde et tradition, Nîmes, Jacqueline Chambon, I989.

58 Je renvoie ici aux réflexions de Lessing sur l'instant fécond que le peintre ou le sculpteur choisit de fixer car il est hautement chargé d'intensité dramatique et qu'il suggère la suite sans la représenter. Voir Gotthold Ephraim Lessing, Laocoon, ou Des frontières respectives de la peinture et de la poésie, Paris, Klincksieck, 20II [première publication: Laokoon, oder über die Grenzen der Malerei und Poesie, Berlin, Christian Friedrich Voss, i766].

59 Voir Marcel Mauss, Essai surle don. Forme et raison de l'échange dans les sociétés archaïques, Paris, PUF, 2007 [première publication: L'Année sociologique, seconde série, I923-1924].

60 Voir notamment Livio Belloï, Le Regard retourné. Aspects du cinéma des premiers temps, Québec/Paris, Nota Bene/ Méridiens Klincksieck, 200I.

61 Voir Adrian Brunel, «Experiments in Ultra-Cheap Cinematography», Close $U p$, vol. $3, \mathrm{n}^{\circ} 4$, octobre i928, pp. 43-46. 